\title{
Decoding bug chatter to fight infections
}

\author{
Nina Konstantinidou \\ Department of Microbiology, UCC
}

\begin{abstract}
"Safe care saves lives and money. Adverse events like high levels of infections, blood clots or falls in hospitals, emergency readmissions and pressure sores cost billions. There is a serious human cost also, with patients ending up injured or even dead. Most are avoidable with the right care" (Lansley A, The Observer 2010).
\end{abstract}

\section{Fungal infections}

We usually link infections with bacteria. However, more than half of the world's population is suffering due to the infections caused by microscopic organisms called fungi. These conditions range from the mildest athlete's foot to life threatening meningitis. Despite diversity, only a few patients are aware that their disease is correlated with fungal agents.

Nowadays, dangerous fungi are becoming more common. The incidence of disease caused by these microscopic bugs has increased at an alarming rate and will double in the coming years. Annually, fungi are responsible for millions of deaths and billions of expenditure. High fungal infection rates are mostly related to the growing population of patients with weakened immune systems. Modern medical practices including chemotherapy, antibiotics and drugs that suppress our defence mechanisms make the cancer patients and transplant recipients ideal targets for fungal attack. Additionally, fungi thrive in hospitals and medical devices. These virulent agents remain a leading cause of infections in patients with urinary catheters and breathing ventilators due to hospital-related drug resistance. These infections often involve several types of microbes but the main agent responsible for serious problems is an elusive Candida. There are over 20 different Candida species but Candida albicans is a key player.

\section{Candida albicans}

In absence of bacteria, fungi like Candida can grow exponentially. Candida albicans is a well-characterised microscopic fungus which displays two modes of growth: yeast (single cellular) and hyphae (brunching filaments). It lives peacefully in the mouth, gut, vagina and skin without compromising person's health. However, Candida infections occur when fungi find the opportunity to increase their population. Hence, C. albicans is an opportunistic germ which mainly causes problems when the environment in the body becomes suitable for the bug to grow and spread. This can happen when a patient takes antibiotics 
and kills the bacteria that compete with fungi for food. Thus, Candida multiplies quickly because the competitor bacteria are killed. For instance, during antibiotic treatment, mucosal infections of genitals are quite common in females, whereas babies, denture wearers and asthma patients develop oral thrush due to fungal agents. However, clinical evidence suggests that these nasty superficial infections are often self-limiting once antibiotic therapy is completed.

\section{Superbugs}

Doctors are now concerned about more serious fungal infections with alarmingly high death rates. Scientists are also warning that widespread chemotherapy treatments and the increased use of drugs which suppress our immune defence can cause deadly fungal diseases. Usually, fungal related deaths are caused by one of four superbugs: Candida, Pneumocystis, Cryptococcus or Aspergillus. Given the global hospital-related blood infections, Candida species are the most common killers that infect approximately half million people annually. C. albicans is a microscopic superfungus which mostly appears as a single cellular yeast. However, it can transform to a more complex form and develop drug-resistant structures. The drug resistance of the problematic $\mathrm{C}$. albicans is linked to its protective structures called "biofilms". Biofilms work like bulletproof vests against antifungal drugs and antibodies of our immune system (Figure 1). Hence, C. albicans remains the most dangerous superbug which can form biofilms to protect itself, communicate with other microbes and cause life threatening infections in humans.

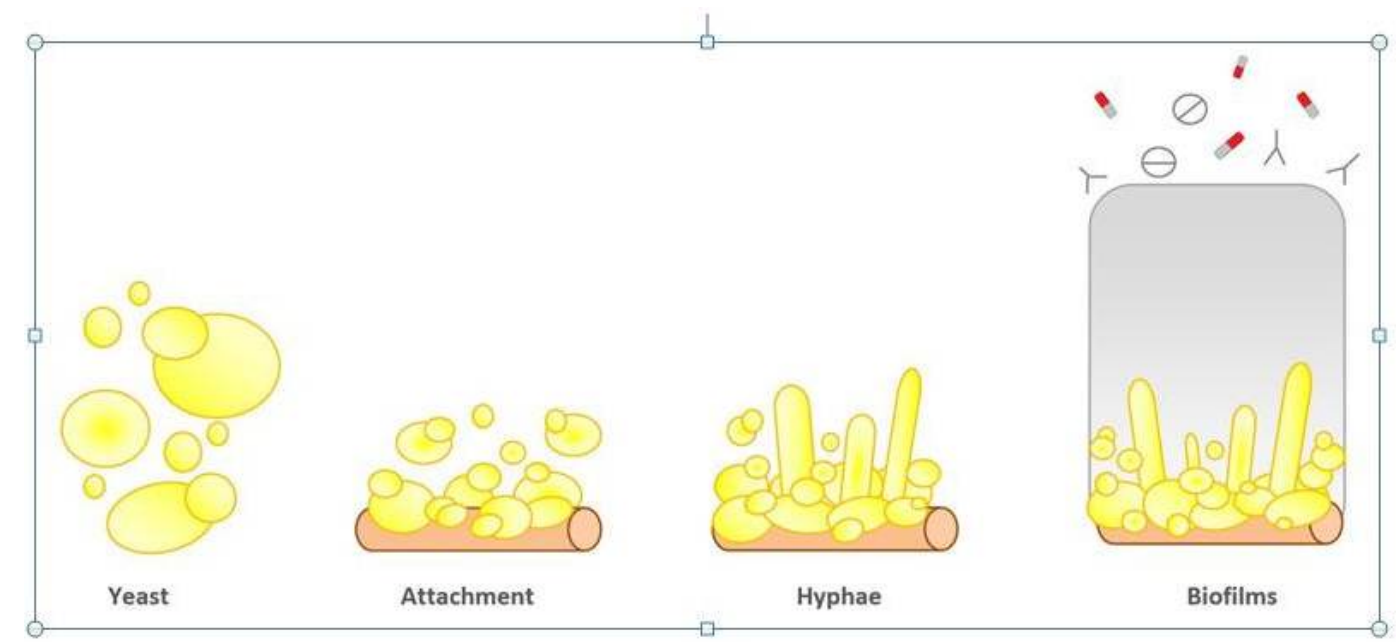

Figure 1: Schematic of fungal biofilm development (adapted from Fox and Nobile, Transcription 2012) 


\section{How bugs communicate}

Large microbial populations need to communicate in order to survive. However, they have no mobile phones or internet chatrooms at their disposal. Instead, they secrete chemical molecules to signal each other and share data, such as their community size. When bacterial populations reach a certain density they take action, which includes secreting toxic chemicals (toxins), competing for food or producing light. This phenomenon is known as "quorum sensing" and is a characteristic of many bacteria including Pseudomonas. Pseudomonas aeruginosa is a common germ which infects cystic fibrosis patients and secretes quorum sensing compounds to communicate.

Normally, germs do not live in tubes on their own but in multiple microbial communities in the wild. Indeed, clinical data suggest that many infections are caused by different microbes that infect us simultaneously. These infections are called "polymicrobial". In a polymicrobial environment, diverse microbes interact with each other developing complex communication networks. Communications between a common bacterium, P. aeruginosa, and the fungus, C. albicans, are intensively studied since they are found together on many infected areas of a human body. The sites frequented by both include burn wounds and the lungs. Additionally, bacteria and fungi form biofilms on plastic medical equipment such as urinary and vascular catheters. Biofilms increase the power of germs by making them practically untreatable. Due to this, recent research has focused on polymicrobial communities and interactions between community members. Communications between bacteria and fungi are well-documented. The best known example is a fungal compound discovered by Alexander Fleming, penicillin, which can kill bacteria. My project focuses on fungal mechanisms (e.g. signalling pathways) which can be modulated via bacterial activity. Interactions between bacteria and fungi can be divided into five main categories:

Physical interactions which occur when bacteria actually attach to a fungal cell surface

Chemical signalling which involves the exchange of secreted chemicals. For example, quorum sensing molecules of bacteria that modulate fungal behaviour

Metabolic by-products that are also used for microbial communications

Environmental changes such as the $\mathrm{pH}$ fluctuation caused by bacteria that influence yeast

Human-host immune system alterations which develop complex networks of interactions 


\section{Objectives of my project}

From microbes to humans, genetic information is inherited via DNA. This nucleic acid is composed of 4 chemical bases, A (Adenine), T (Thymine), G (Guanine) and C (Cytosine) that form DNA sequences. Some regions of DNA sequences, known as genes, contain instructions to encode important biological molecules, proteins, whereas others can regulate behaviour of an organism according to environmental cues.

My project provides the opportunity to gain experience with two scientifically important organisms: yeast and bacteria. Overall, the aim is to identify specific fungal genes that are mostly affected by bacterial signals. Fungi are complex organisms that can sense bacterial signals with different chemical structures and biological properties. Bacterial signals are sensed by fungal cell surface systems termed, cell receptors. The signal is passed through the cell by specific proteins called, kinases. Another group of proteins, transcription factors, activate the target genes that define fungal response to the bacterial signal. Many studies have shown that bacterial signals can inhibit fungal biofilm formation. Hence, the initial objective of my research includes high throughput screening of genetically modified Candida strains. High throughput screening is a scientific method used to identify genes that modulate behaviour of an organism. Each genetically modified Candida strain lacking one protein kinase encoding gene will be tested for biofilm formation in the presence of $P$. aeruginosa molecules. Ideally, the screen will reveal the mutants that are not affected by bacterial chemicals and, in contrast to the wild type, can form biofilms even after application of $P$. aeruginosa secretions. Another aim is to develop a molecular system with the gene of interest and a green florescent protein which will enable a rapid screen of genetically modified Candida strains via fluorescent microscopy.

\section{Primary screen}

Fungi are usually investigated after they are cultured in different growth media. Depending on media properties, the fungi can die, multiply or form colonies, filaments and biofilms. The degree of fungal biofilm formation ability can be measured with several methods. The primary screen of genetically modified fungi was performed via a quantitative biofilm assay. An assay is an important process of a scientific investigation applied to quantify the results of an experiment. Quantification is a fundamental method of counting observations and translating them into numbers. This approach enabled a reliable estimation of fungal biofilms according to their colour. After the addition of bacterial signalling molecules and colouring solutions to the cultures of each Candida mutant, colour intensity of the cultures was measured and quantified. Then, all the measurements were analysed statistically and visualised via comprehensive graphs. Another fundamental strategy in biomedical experiments lies in the comparison of a control with question groups. Hence, 
wild type $C$. albicans was used as a control against mutants in order to observe differential biofilm formation trends. Both wild type and mutants were grown in biofilm inducing media with and without the application of bacterial secretions.

From previous studies, it was known that the wild type Candida is unable to form biofilms in the presence of bacterial chemicals. Hence, any mutant lacking a protein kinase gene that could still form biofilms after the addition of bacterial secretions, was interesting for our study. Primary screen across protein kinase mutants revealed two mutants that could develop biofilms after the addition of bacterial molecules. This indicated that the missing genes could be essential for sensing the mechanism of bacterial signals since mutants lacking these genes were unable to respond to the bacterial chemicals. These findings suggested that the two genes could play a key role in the signalling pathways of $C$. albicans that responds to bacterial chemicals.

\section{Potential benefits of my research}

Millions of people have suffered from fungal infections. While some have felt discomfort because of athlete's foot others have died from meningitis, all caused by microscopic fungi. Moreover, despite the wide use of drugs, fungal infections remain a leading cause of morbidity and mortality in the hospitals. I believe I have found two Candida genes that may play a key role in the signalling pathways of this fungus. This contributes to our understanding of fungal virulence. Hence, my project may have multiple clinical applications. Thus, my findings are critical for the development of effective antifungal therapeutic strategies that can save lives and money.

Nina Konstantinidou is a first year Ph.D. student in the School of Microbiology and part at the HEA-funded Molecular Cell Biology Structured Ph.D. Programme. She is supervised by Dr. John Morrissey whom she would like to thank for his continuous support. Nina also thanks Prof. Clarissa Nobile for her permission to cite an adapted schematic of $C$. albicans biofilm development. 